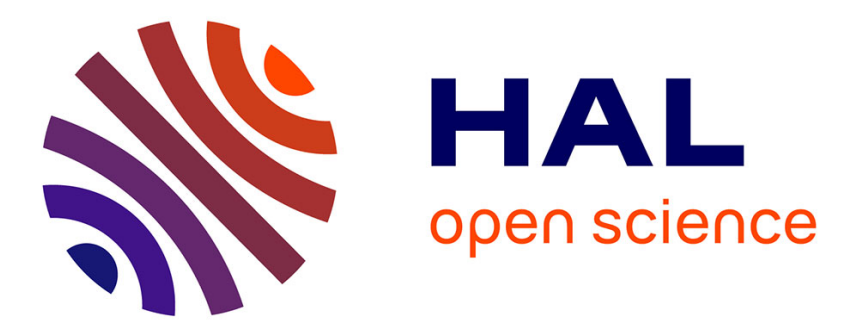

\title{
Electromagnetic characterization of metamaterials in the centimetric frequency band using an original adjustable height stripline
}

\author{
Alexis Chevalier, Sandra Gomez, Patrick Queffelec
}

\section{- To cite this version:}

Alexis Chevalier, Sandra Gomez, Patrick Queffelec. Electromagnetic characterization of metamaterials in the centimetric frequency band using an original adjustable height stripline. Journal of Applied Physics, 2014, Vol. 116, pp.124901. 10.1063/1.4828876 . hal-01068637

\section{HAL Id: hal-01068637 \\ https://hal.univ-brest.fr/hal-01068637}

Submitted on 26 Sep 2014

HAL is a multi-disciplinary open access archive for the deposit and dissemination of scientific research documents, whether they are published or not. The documents may come from teaching and research institutions in France or abroad, or from public or private research centers.
L'archive ouverte pluridisciplinaire HAL, est destinée au dépôt et à la diffusion de documents scientifiques de niveau recherche, publiés ou non, émanant des établissements d'enseignement et de recherche français ou étrangers, des laboratoires publics ou privés. 


\title{
Electromagnetic characterization of metamaterials in the centimetric frequency band using an original adjustable height stripline
}

\author{
A. Chevalier, S. Gomez, and P. Queffelec \\ Laboratoire des Sciences et Techniques de l' information, de la Communication et de la Connaissance \\ (Lab-STICC) UMR CNRS 6285, Université de Bretagne Occidentale - UBO, CS 93837, 29238 Brest, France
}

(Received 27 June 2014; accepted 12 September 2014; published online 23 September 2014)

\begin{abstract}
We propose a broadband measurement method for measuring the electromagnetic (EM) properties of metamaterials in the $1-6 \mathrm{GHz}$ frequency range. For this experimental technique, an original adjustable height strip transmission-line was developed that makes it possible to characterize metamaterials with different volumes and different numbers of unit cells. Therefore, it was possible to assess the influence of the number of unit cells in a sample on the measured constitutive parameters (complex permittivity and permeability). This study enabled us to define the criterion of the representative elementary number of cells. This criterion must be satisfied in practice in order to determine the microwave behavior for the sample under test in as close as possible to an infinite medium, whose EM properties can only be determined from simulations. Experimental and theoretical results for split ring resonator-type metamaterials are presented, compared, and discussed. (C) 2014 AIP Publishing LLC. [http://dx.doi.org/10.1063/1.4896385]
\end{abstract}

\section{INTRODUCTION}

Metamaterials ${ }^{1}$ have been widely studied in recent years because of their unconventional electromagnetic (EM) properties. Most metamaterials are made of metallic inclusions that are specifically designed to have desirable dynamic properties. However, the heterogeneous, anisotropic, and strongly dispersive characters of metamaterial dynamic behavior make it difficult to measure their EM properties. This step is nevertheless essential to validate theoretical models and to test samples before using them in practical applications.

Once the homogenization ${ }^{2}$ of the studied material can be performed at the intended frequencies (the magnitude of the heterogeneities remains smaller than the wavelength), the design of an EM characterization method adapted to metamaterials raises the fundamental question of to what extent measurable parameters, such as impedance or scattering parameters (S-parameters), can be representative of the metamaterial physical parameters so as to determine the effective permittivity and permeability tensor $\left(\varepsilon_{\mathrm{eff}}, \mu_{\mathrm{eff}}\right)$. To guarantee this representativeness, the experimental method must satisfy two conditions: first, the excitation of a minimal number of metal inclusions (unit cells (UC)) present in the sample tested and second, the homogeneity for both magnitude and direction of the incident EM wave over the whole volume of the sample.

The free space reflection/transmission method ${ }^{3,4}$ addresses these problems; as long as the sample is large enough in relation to the wavelength, so to avoid edge effects. This last condition can easily be achieved in the millimeter wave range, but becomes prohibitive in the centimeter wave range because it leads to sample sizes larger than several tens of centimeters. To overcome this issue, we proposed a characterization method based on a stripline fixture, published in a previous paper. ${ }^{5}$ This work showed that the stripline method is well suited to the characterization of metamaterials in the centimeter wave range. The main advantage of this method is that all inclusions are uniformly excited by a constant EM field in terms of direction and magnitude. However, the fixed geometry of the characterization fixture limits the measurements to samples with fixed dimensions.

In the present work, we propose a new measurement method based on an original stripline fixture with adjustable ground plane positions. This new measurement fixture makes it possible to study the influence of the number of unit cells composing the sample under test in order to evaluate interactions between adjacent inclusions.

In the first part of this paper, we present the principle of the method worked out for EM characterization of metamaterials in the centimeter wave range. The geometry and dimensions of the experimental fixture are specified, which satisfy the criteria listed above. The EM analysis used in the data processing program to relate the EM properties of the sample to the measured S-parameters is also presented.

In the second part of this paper, we describe the specific calibration procedure and the experimental protocol necessary to solve problems related to the adjustable height of the measurement fixture. Then, we present, for Split Ring Resonator (SRR)-type ${ }^{6} \mathrm{UC}$, the comparison between experimental responses of metamaterials and EM simulations.

Finally, in the third part of this paper, we present our study on the influence of UC number in the test sample on the measured constitutive parameters $\left(\varepsilon_{\mathrm{eff}}, \mu_{\mathrm{eff}}\right)$. We specify the experimental conditions necessary to determine the asymptotic behavior of the measured sample that occurs when EM properties converge through values corresponding to those of an infinite virtual medium.

\section{DESCRIPTION OF THE MEASUREMENT METHOD}

The experimental technique we developed uses an original symmetrical stripline fixture with variable position ground planes. This particular configuration allows the height of the cell to be adjusted in order to measure materials of 


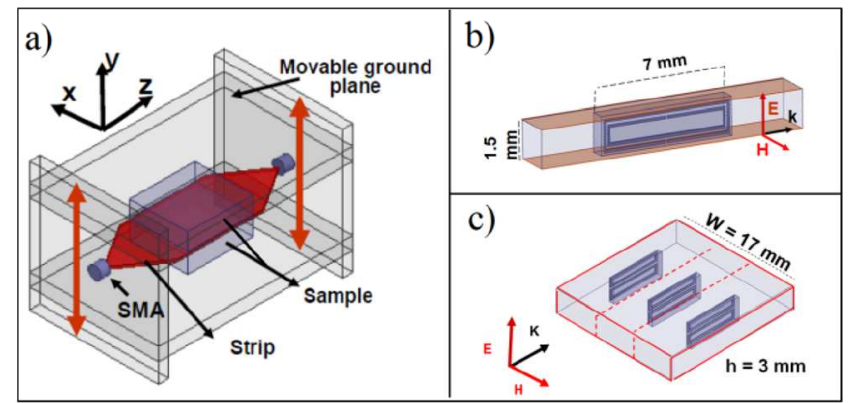

FIG. 1. (a) Measurement stripline fixture. (b) SRR unit cell. (c) Design of the split-ring resonator metamaterial sample (example of three slabs with two unit cells).

different sizes. Two identical pieces of sample are placed below and above the central strip as shown in Fig. 1. For practical manufacturing purposes, we chose to work on SRRtype metamaterial. The metamaterial unit cell was designed to allow an increase in the number of inclusions according to the height, transversal, and longitudinal directions.

Fig. 1(a) presents the concept of the measurement stripline fixture; ground planes can move in the y direction in order to adjust the total height $2 h$ of the stripline. The width $w$ of the strip is set at $17 \mathrm{~mm}$ in order to reject the cut-off frequency of the first higher mode $\mathrm{TE}_{10}$ above $6 \mathrm{GHz}$ whatever the height $h$ in the operating range. Fig. 1(b) presents the design of the Split-Ring Resonator UC we chose for this study. Elongated square ring and split dimensions of the UC were chosen in order to present a theoretical resonant dynamic response occurring in the operating frequency band [1-6 $\mathrm{GHz}]$. Outer $\left(6.75 \times 1.25 \mathrm{~mm}^{2}\right)$ and inner $\left(6.3 \times 0.8 \mathrm{~mm}^{2}\right)$ rectangular loops are separated by a $125-\mu \mathrm{m}$ thick gap. Copper metallization (thickness $17 \mu \mathrm{m}$ and width $100 \mu \mathrm{m}$ ) was etched on a $635-\mu \mathrm{m}$ thick dielectric substrate AR1000 ${ }^{\circ}$ with permittivity equal to 10 and loss tangent equal to 0.003 . The split was set at $100 \mu \mathrm{m}$. Unit cells were then arranged in several ways in a sample holder and inserted into the measurement fixture. Fig. 1(c) presents an example of an arrangement with $3 \mathrm{UC}$ in the transversal direction (x), $2 \mathrm{UC}$ in the height direction (y), and $1 \mathrm{UC}$ in the propagation direction (z).

The EM field distribution of the dominant TEM propagation mode in the central area satisfies the requirements for metamaterial characterization: The electric field is parallel to the metallic inclusions, the magnetic field is perpendicular to them and the magnitude is constant over the whole volume of the sample (Fig. 2(a)).

As detailed in previous work, ${ }^{5}$ we used a theoretical approach based on the transmission line theory to retrieve the complex constitutive parameters $\left(\varepsilon_{\mathrm{eff}}, \mu_{\mathrm{eff}}\right)$ of the metamaterial placed inside the measurement fixture from the measured S-parameters.

Assuming, first, that the most of the EM energy is concentrated in the region located between the central metallic strip and the ground plane and, second, that the width of the metamaterial sample does not exceed the width of the strip, for the EM analysis, we consider only the region located between the strip and the ground plane. This transversal section, which only matches one half cross section due to the

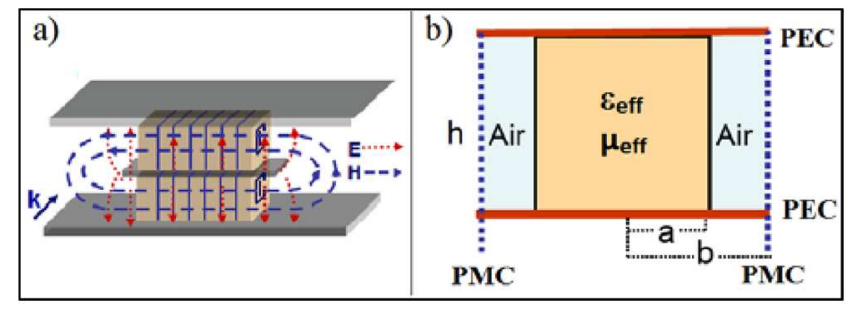

FIG. 2. (a) Electromagnetic field pattern in the stripline. (b) Equivalent representation of the line cross section for the EM analysis.

symmetry of the structure, is an equivalent to a two conductor transmission line as depicted in Fig. 2(b). Perfect electric conductor (PEC) walls are used for the central strip and the ground plane, while perfect magnetic conductor (PMC) walls are used as boundary conditions at the edge of the strip, considering the symmetry of the field pattern related to the dominant TEM mode. Expressions of the effective permittivity and permeability of the metamaterial are derived from the $S$ parameters using a method comparable with the Nicolson Ross Weir procedure ${ }^{7,8}$

$$
\begin{gathered}
\mu_{e f f}=\frac{a Z 2 \gamma(1+R)}{h \mu_{o} \omega(1+R)-(b-a) Z 2 \gamma(1+R)}, \\
\varepsilon_{e f f}=\frac{[a+(b-a) \mu] \gamma^{2}}{a \mu_{o} \varepsilon_{o} \mu \omega^{2}}+\frac{(b-a)}{a},
\end{gathered}
$$

where $a$ is the half-width of the sample, $b$ is the half-width of the strip, $\mathrm{Z}$ is the characteristic impedance of the line (dependent on the height $h$ ), $\gamma$ is the propagation constant, and $\omega$ is the angular frequency. $\mathrm{R}$ is related to the measured $\mathrm{S}$ parameters as follows:

$$
\begin{gathered}
R=K_{1} \pm \sqrt{K_{1}^{2}-\frac{S_{11}}{S_{22}}}, \\
K_{1}=\frac{S_{11} S_{22}-S_{12} S_{21}+1}{2 S_{22}}, \\
\gamma=\frac{j}{d} \ln \left(\frac{S_{21}}{1-R S_{22}}\right) .
\end{gathered}
$$

In order to validate the data processing program related to the measurement fixture, we performed full-wave simulations with commercial ANSYS HFSS $\left.{ }^{(}\right)$software. The measurement configuration presented in Fig. 1 has been exactly reproduced; electrical properties for $\operatorname{AR} 1000^{\circ}\left(\varepsilon_{\mathbf{r}}=10\right.$; $\operatorname{tg} \delta=0.003)$ and copper $\left(\sigma=58.10^{6} \mathrm{~S} / \mathrm{m}\right)$ were specified to obtain the simulated S-parameters of the metamaterial put in the test fixture. Then Eqs. (1) and (2) were used to extract the simulated effective permittivity and permeability $\left(\varepsilon_{\mathrm{eff}}\right.$, $\mu_{\text {eff }}$ ) of the metamaterial.

\section{EXPERIMENTAL SETUP}

The measurement fixture (Fig. 3) is built in aluminum. Two radio frequency subminiature version A (SMA) connectors are used to connect the fixture to the junction cables of the network analyzer. The height $h$ between the two ground 


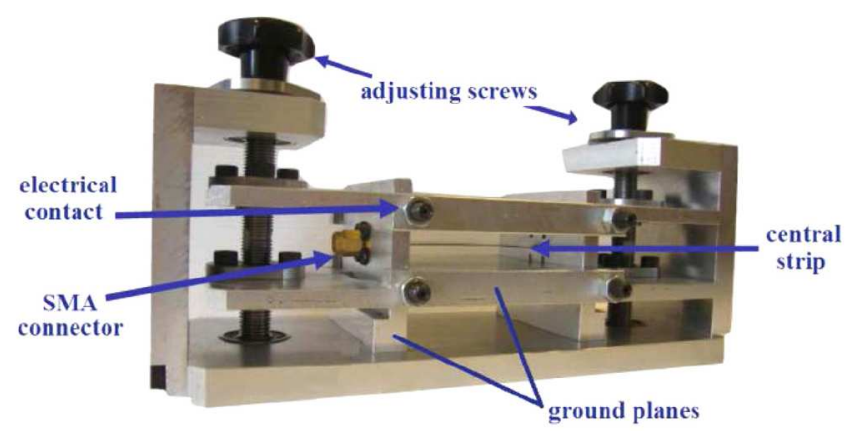

FIG. 3. Adjustable stripline fixture prototype as metamaterial measurement cell.

planes of the prototype measurement cell can be varied from $1 \mathrm{~mm}$ to $10 \mathrm{~mm}$ by adjusting two control screws as shown in Fig. 3. The strip length is $60 \mathrm{~mm}$, its width is $17 \mathrm{~mm}$, and its thickness is $300 \mu \mathrm{m}$.

As the height of the fixture is adjusted, the characteristic impedance $Z$ changes from $10.4 \Omega(h=1 \mathrm{~mm})$ to $72 \Omega$ ( $h=10 \mathrm{~mm}$ ) according to the Wheeler expression. ${ }^{9}$ Therefore, the non-50-Ohm impedance and its change according to the height require a specific calibration procedure. The first step of the experimental procedure consists of calibrating the network analyzer in order to correct systematic errors. We perform a standard "Short Open Load Thru" calibration leading to reference planes located at the ends of cables in the SMA connector planes.

The second step consists of calibrating the empty measurement cell in order to correct imperfections due to finite conductivity and impedance mismatching caused by the adjustable height. During this step, the height of the empty measurement fixture is adjusted to that of the sample under test. Furthermore, to calculate the permittivity and permeability of the sample $\left(\varepsilon_{\mathrm{eff}}, \mu_{\mathrm{eff}}\right)$ from Eqs. (1) and (2), we have to perform a displacement of the reference planes for the phase measurements from connector plane to sample plane. This step of de-embedding is performed using a conventional cascade-based method, ${ }^{10}$ in which each section of the fixture is represented by a transfer $\mathrm{T}$ matrix. ${ }^{11,12}$ Thus, in the propagation direction, we can identify the following sections: a 50Ohm coaxial section at the SMA connectors followed by a "taper" acting as the coaxial to stripline transition and finally the stripline section.

The S-parameters are measured in the frequency band [1-6 GHz] using an Agilent 8753ES Vector Network Analyzer. Figure 4 presents the comparison between theoretical and measured S-parameters of the empty fixture for $h$ equal to $6 \mathrm{~mm}(Z=27.4 \Omega)$. We show that, as a result of the successful de-embedding process, the theoretical response fits perfectly to the measured one.

The final step then consists of measuring the Sparameters of the fixture loaded with the sample under test. The de-embedding uses the same presented procedure in order to compare measured and simulated S-parameters in the sample's reference planes and to retrieve constitutive parameters $\left(\varepsilon_{\text {eff }}, \mu_{\text {eff }}\right)$ from Eqs. (1) and (2). Several reference samples of well-known dielectric materials were firstly tested to evaluate the accuracy of the measurement method. The total uncertainty due to calibration procedure, measurement uncertainties, and assumptions of the retrieving method was found less than $5 \%$.

Then, as an application to metamaterial of the measurement method, Fig. 5 shows the HFSS-simulated and measured S-parameters for the metamaterial sample configuration depicted in Fig. 1(c). We can clearly identify the effect of the metamaterial represented by attenuation peaks in transmission $S_{21}$ and reflection $S_{11}$ coefficients.
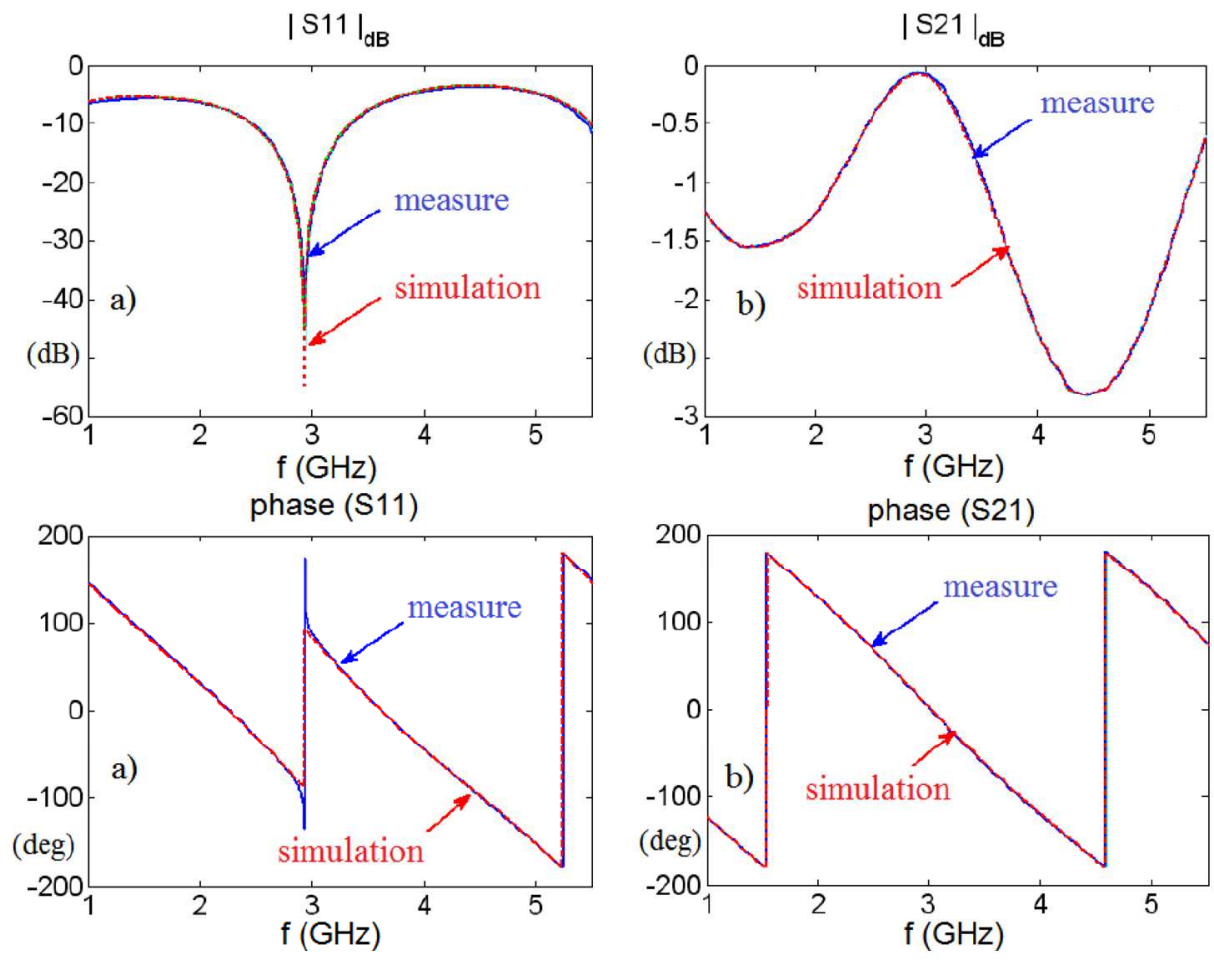

FIG. 4. Comparison between theoretical (red dashed line) and measured (blue solid line) S-parameter of the empty fixture: reflection (a) and transmission (b) coefficients. 

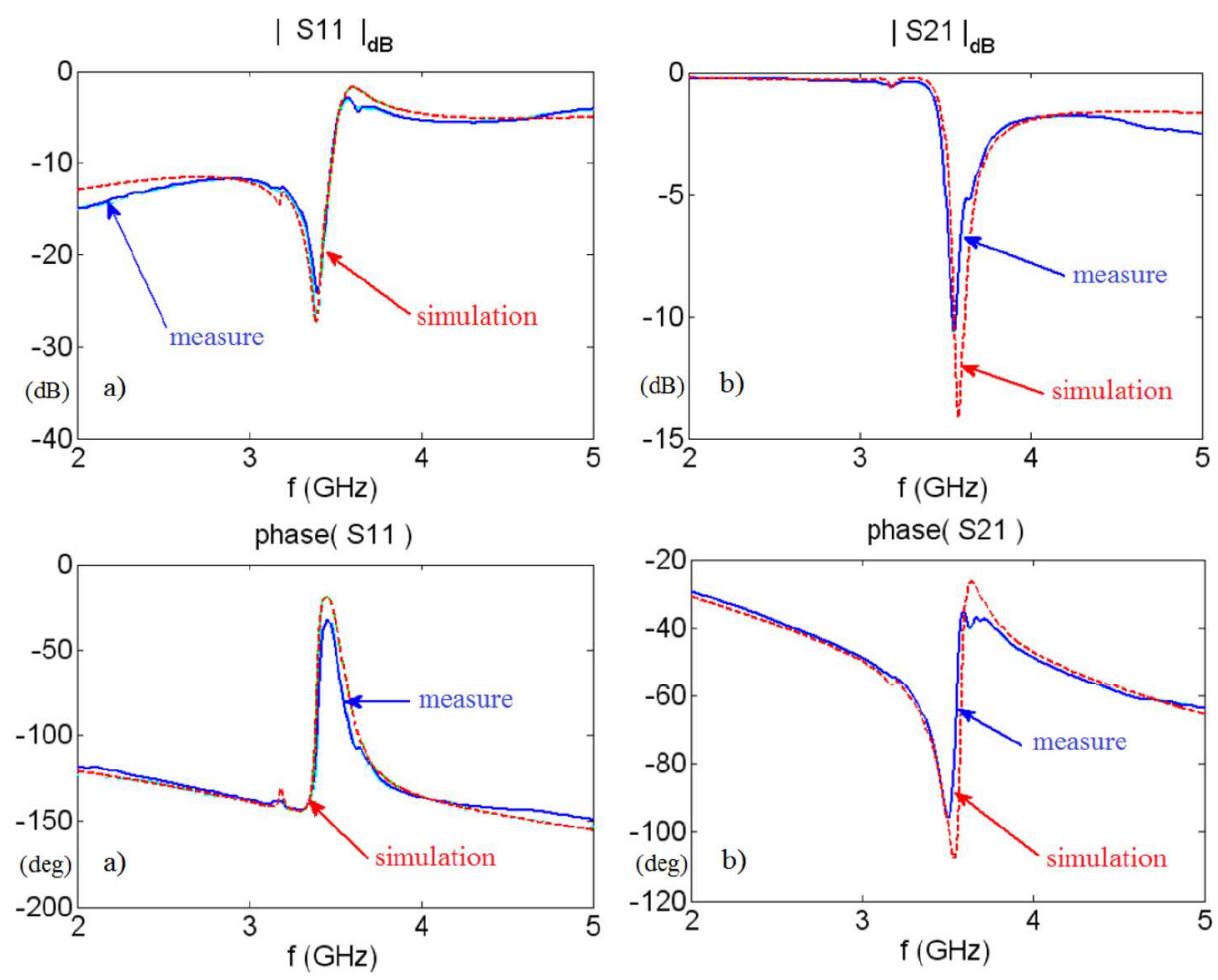

FIG. 5. Comparison between simulated (red dashed line) and measured (blue solid line) S-parameter of the fixture loaded with metamaterial: reflection (a) and transmission (b) coefficients.

Fig. 6 shows the complex permittivity and permeability $\left(\varepsilon_{\text {eff }}, \mu_{\text {eff }}\right)$ spectra (real and imaginary parts) of the sample retrieved from the measured S-parameters compared with spectra deduced from the simulated S-parameters obtained with HFSS. A possible dispersion of the dielectric properties of the SRR substrate, which should be very low at operating frequencies, is not taken into account during the simulations.

The expected resonant behavior of this SRR-type metamaterial clearly appears in both measured and calculated spectra. We observe a resonance frequency close to $3.5 \mathrm{GHz}$ and a negative real part of the permeability between 3.5 and $3.7 \mathrm{GHz}$. The electrical response is also resonant, exhibiting a positive value all over the entire exploited frequency band. A good agreement is observed between measured and simulated data.

Note that thanks to the movable ground planes, air gaps, first between the ground plane and the sample and, secondly between the central strip and the sample are minimized. However in practice, there may remain small air gaps. These gaps were valued close at $30 \mu \mathrm{m}$. Their effects on the permittivity were then corrected using the Wiener's law as presented in previous work, ${ }^{5}$ while effects on the permeability were considered insignificant due to the magnetic field pattern.

\section{INFLUENCE OF THE UNIT CELLS NUMBER}

In order to highlight the effects of increasing the number of unit cells in the metamaterial, measurements and simulations were performed increasing the number of UC in all three directions.

In the height direction (y axis), the number of UC was increased from 1 to 3 . For each height, the complete
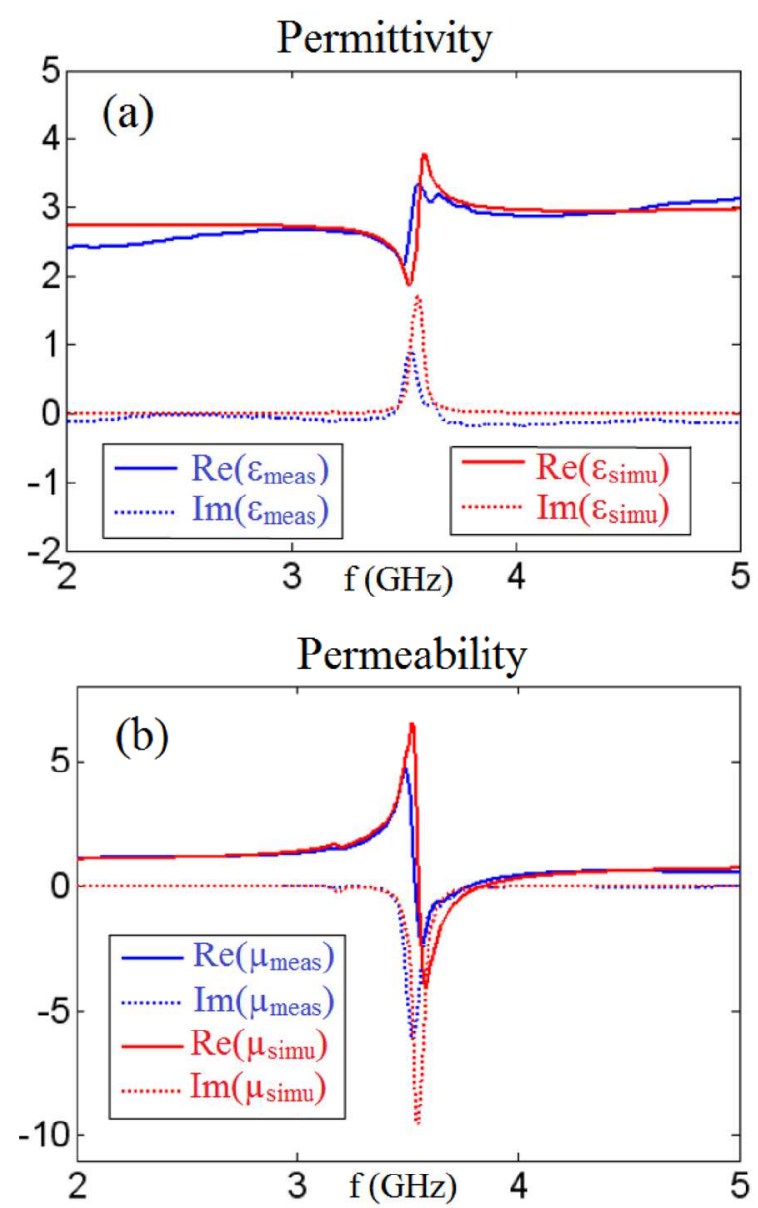

FIG. 6. Real (solid line) and imaginary (dashed line) parts of the permittivity (a) and permeahility (b) spectra retrieved from simulated (red) and measured (blue) S-parameters. 
measurement and calibration procedures were performed in order to extract the constitutive parameters $\left(\varepsilon_{\text {eff }}, \mu_{\text {eff }}\right)$ of the metamaterial regardless of the stripline impedance.

Fig. 7 first presents the UC configuration in the metamaterial sample to be characterized and then shows the permittivity and permeability spectra retrieved from the simulated (HFSS software) and measured S-parameters. In the spectra retrieved from simulations, we can observe that all frequency responses are identical and the resonance frequency does not shift when the number of UC increases.
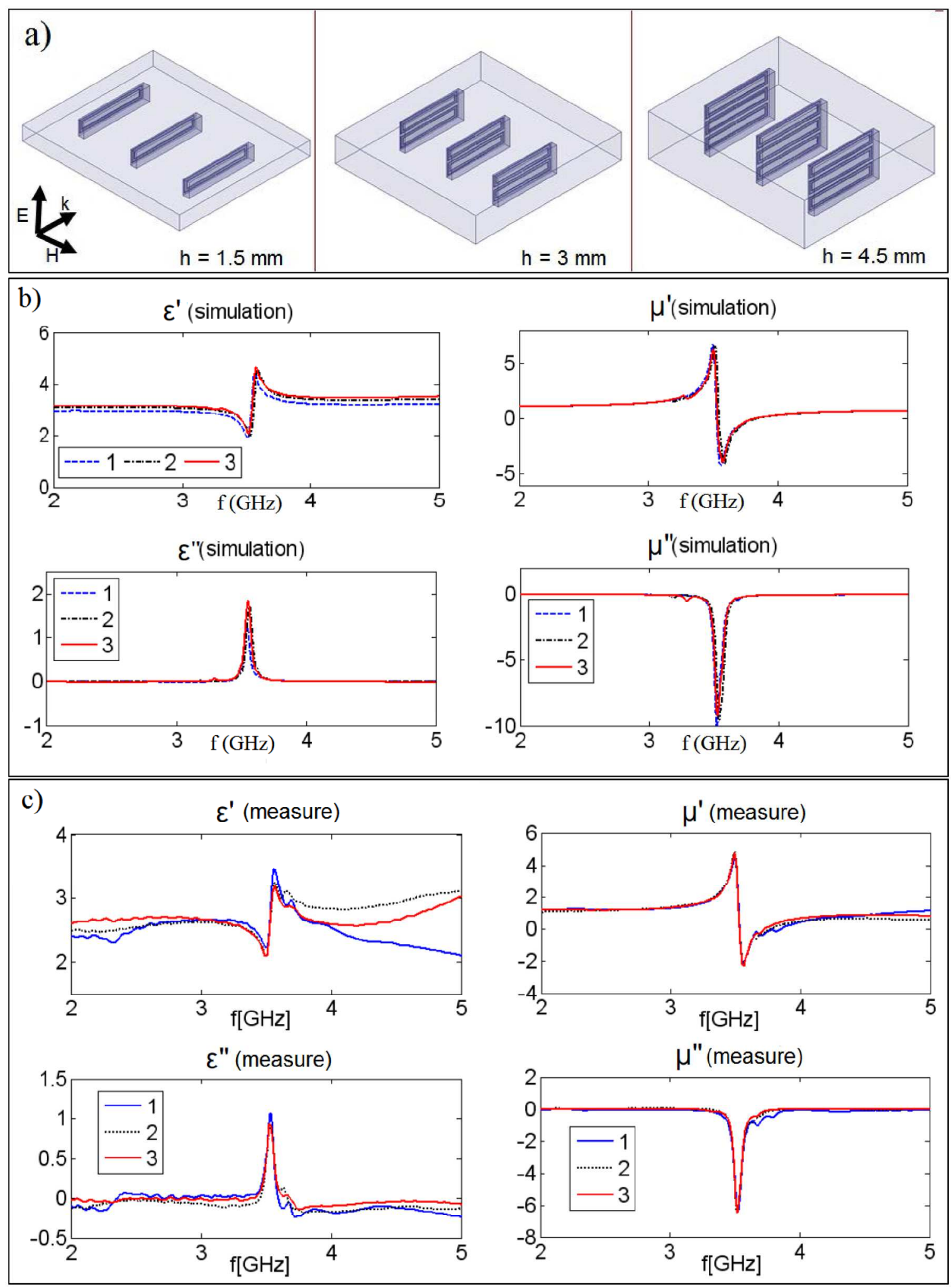

FIG. 7. Influence of the number of unit cells (UC) in the (y) direction. (a) UC configurations. Permittivity and permeability spectra retrieved from (b) simulated and (c) measured S-parameters. 
These results show that the number of UC in the height direction (y) does not influence EM properties for our SRR metamaterial design. This behavior can be attributed to the small interactions that can occur in the E-field direction according to the small thickness of metallization. Although noisy measurements appear in configurations with one and two UC, arising from calibration difficulties when the magnitude of the measured signal is weak; very good agreement is observed between experimental results and simulation. Our conclusion on this part of the study is that the asymptotic behavior is reached with only $1 \mathrm{UC}$, meaning that a sample with one UC can represent the response of an infinite sample in the height direction.

In the propagation direction ( $\mathrm{z}$ axis), we increased the number of UC from 1 to 3 as shown in Fig. 8(a). The measured frequency responses of permittivity and permeability (Fig. 8(b)) are complex and reveal several resonance peaks. As the number of UC increases, we observe the emergence of new resonances in the lower part of the exploited frequency range. Interactions between UC strongly alter the frequency spectra. Moreover, we observe a dimensional resonance close to $5 \mathrm{GHz}$ when the sample length equals one half wavelength. Finally, in this direction, it was not possible to observe an asymptotic behavior of the measured effective permittivity and permeability.

Unfortunately, we could not obtain accurate HFSS simulations for this sample's configuration due to numerical issues and meshing difficulties. As shown by the experimental curves, the complexity of the electromagnetic response of the metamaterial causes the simulation to fail. A very small mesh would be required to obtain accurate simulation but would lead to an excessive calculation time. This demonstrates the interest of our measurement method, which allows the experimental EM characterization of metamaterials that are very difficult to simulate.

In the transversal direction ( $\mathrm{x}$ axis), the number of UC was increased from 1 to 6, as illustrated in Fig. 9(a) (for purposes of clarity, only the first three rows are shown on the figure). Inclusions are positioned in order to present constant spacing in the transversal direction. Because there was no change in the permeability response, only the permittivity response is represented.

We can observe in Fig. 9(b) how the permittivity of the metamaterial sample retrieved from the S-parameters simulated changes as a function of the number of UC. Due to interactions between metallic inclusions, the permittivity resonance frequency (plotted by the maximum of the imaginary part) shifts to lower frequencies as the UC number increases. However, Fig. 10 shows that the behavior of the structure converges and the resonant frequency tends toward the value determined for the same metamaterial but with infinite dimensions. Figure 9(b) shows the imaginary part of the permittivity retrieved from the measured S-parameters. Although noisy measurements still appear in configurations with one and two UC and small discrepancies are noticed in frequency values, very good agreement is observed between the experimental and simulated behavior of the resonance frequency. Nevertheless, we can notice in Figure 10 that the simulated resonance frequency values are higher than the experimental ones for configurations with few UC. This highlights the difficulty to perfectly simulate the actual structure taking into account dimensions irregularities, misalignment,
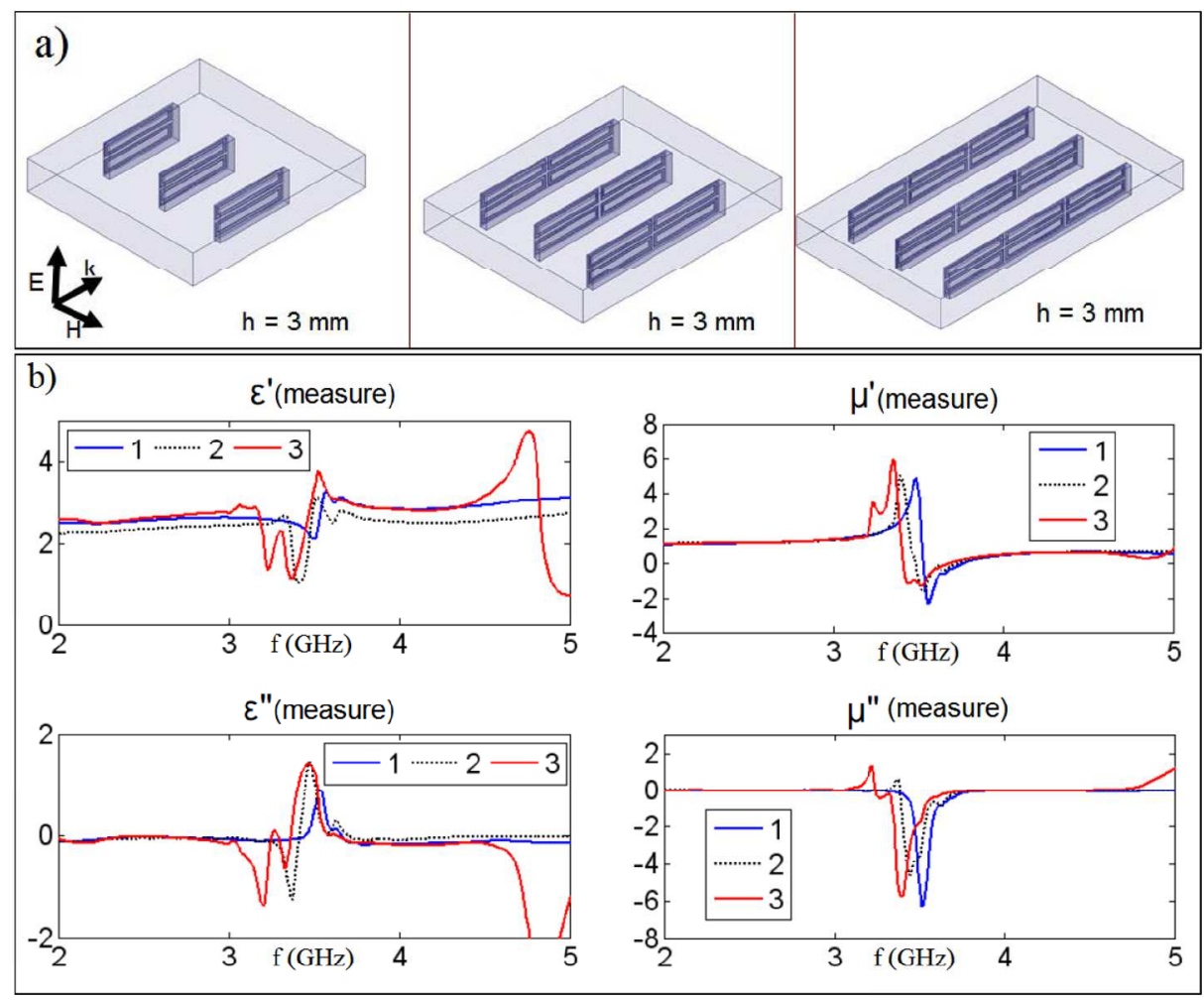

FIG. 8. Influence of the number of unit cell in the (z) direction: (a) UC configurations. Measured permittivity (b) and permeability spectra. 


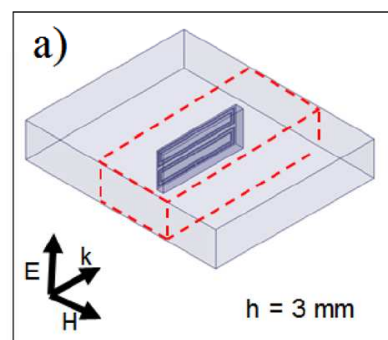

b) $\quad \varepsilon^{\prime}$ (simulation)
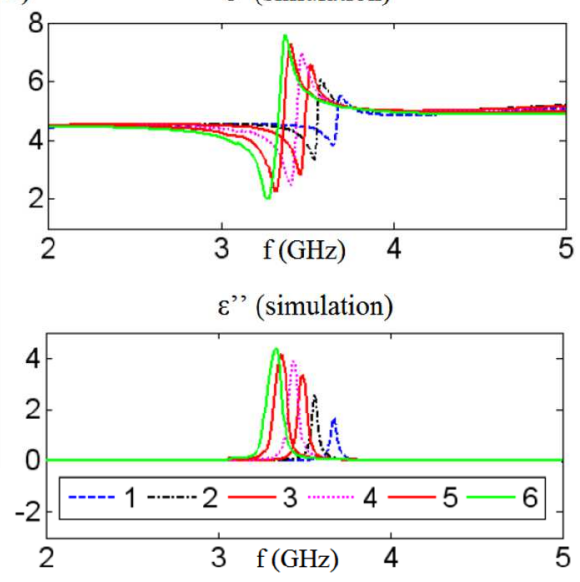
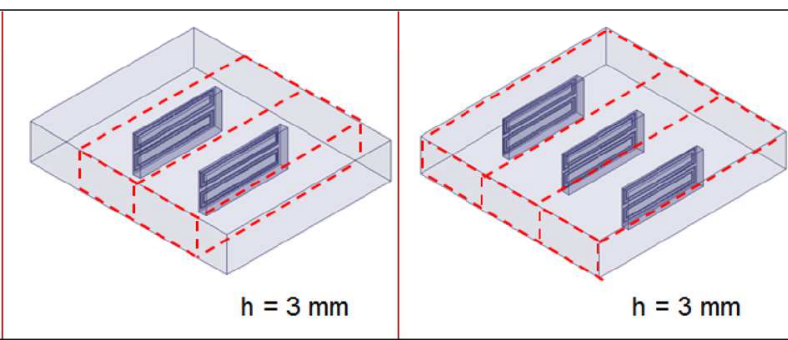

$\varepsilon^{\prime}$ (measure)
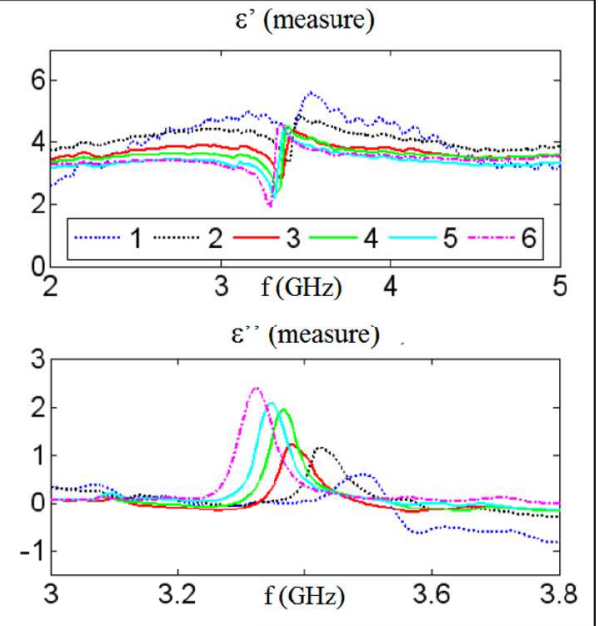

FIG. 9. Influence of the number of unit cell (UC) in the (x) direction. (a) UC configurations. (b) Measured and simulated permittivity spectra.
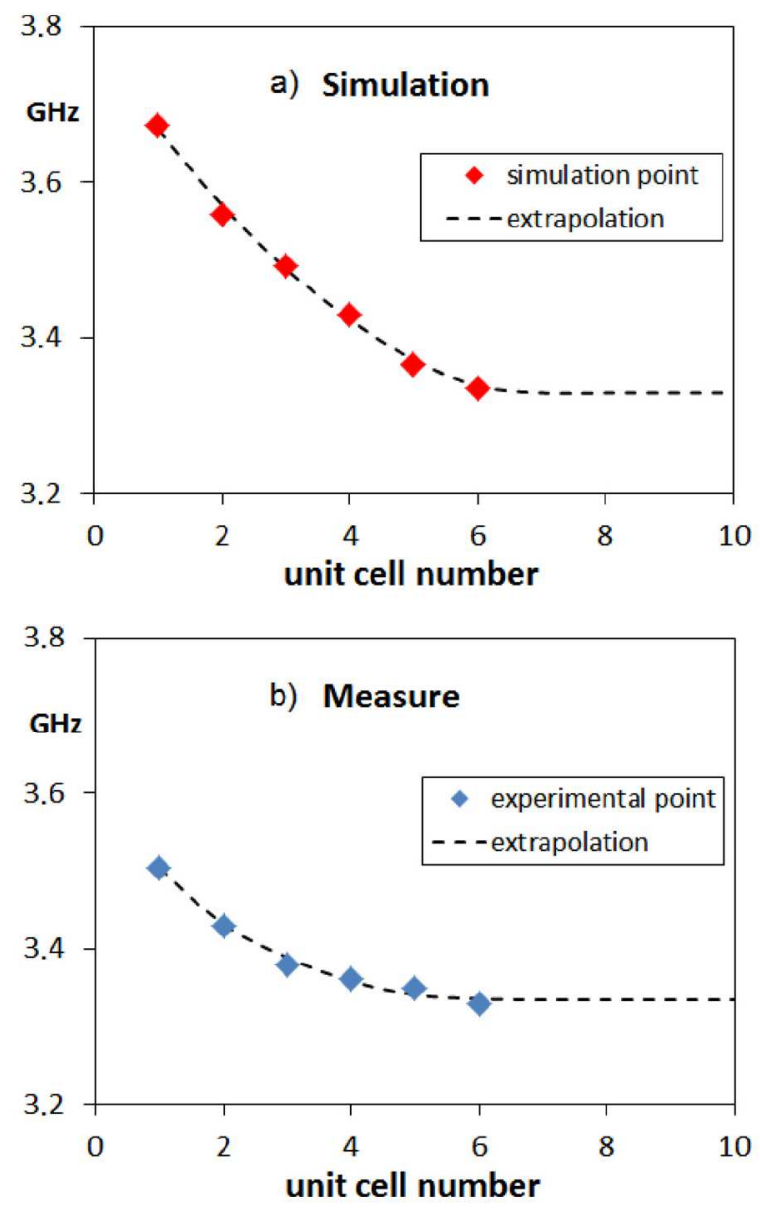

FIG. 10. Variation of the permittivity resonant frequency as a function of the number of UC (a) simulation, (b) measure. or inaccurate value of metallization's conductivity. However, as the number of UC increases, imperfections become less significant and discrepancies vanish, so both measure and simulation converge to the same resonance frequency value.

This experimental demonstration enables us to define the representative elementary number of UC as close to six for this metamaterial design. Our conclusion on this part of the study is that a sample with six UC can represent the response of a metamaterial of infinite dimension in the transverse direction.

\section{CONCLUSION}

In this paper, we have presented a new experimental method for characterizing the EM behavior of metamaterials in the centimeter wave range [1-6 GHz]. The adjustable height stripline fixture makes it possible to measure metamaterials made up of different numbers of unit cells (UC) in the three directions $\mathrm{x}, \mathrm{y}$, and $\mathrm{z}$. Thus, it is possible to evaluate the effect of interactions between adjacent metallic inclusions. We showed that, for the SRR-type metamaterials used in this study, the frequency responses depend on the number of UC in the transversal and propagation directions, but not in the height direction. Moreover, as the number of UC increases, the theoretical convergence behavior was experimentally proven. Thus, we found that, in the transverse direction, a sample containing $6 \mathrm{UC}$ could represent an infinite sample. Very good agreements between experiments and simulations were found for the tested SRR-type metamaterial, confirming the relevance of the experimental method and test fixture developed. Future work based on 
this study could test other designs or metamaterial sample configurations in order to examine interactions in the height direction.

${ }^{1}$ N. Engheta and R. Ziolkowski, Metamaterials: Physics and Engineering Explorations (Wiley-IEEE, 2006).

${ }^{2}$ T. Cui, D. Smith, and R. Liu, Metamaterials: Theory, Design, and Applications (Springer, 2009).

${ }^{3}$ R. Greegor, C. Parazzoli, and K. Li, Opt. Express 11, 688 (2003).

${ }^{4}$ Y. Hollander and R. Shavit, Microwaves Antennas Propag. 5, 84 (2011).
${ }^{5}$ S. Gómez, P. Quéffélec, A. Chevalier, A. C. Tarot, and A. Sharaiha, J. Appl. Phys. 113, 024912 (2013).

${ }^{6}$ J. B. Pendry, A. J. Holden, D. J. Robbins, and W. J. Stewart, IEEE Trans, Microwave Theory Tech. 47, 2075 (1999).

${ }^{7}$ A. Nicolson and G. Ross, IEEE Trans. Instrum. Meas. 19, 377 (1970).

${ }^{8}$ S. Mallégol and P. Quéffélec, IEEE Trans. Microwave Theory Tech. 54, 1065 (2006).

${ }^{9}$ H. Wheeler, IEEE Trans. Microwave Theory Tech. 26, 866 (1978).

${ }^{10}$ A. Chevalier, J. Cortes, J. Lezaca, and P. Queffelec, J. Appl. Phys. 114, 174904 (2013).

${ }^{11}$ D. Pozar, Microwave Engineering (Wiley, 2009).

${ }^{12}$ Agilent Technologies, Application Note 1364-1, 2004. 